International Journal of Pure and Applied Mathematics

Volume $96 \quad$ No. $3 \quad 2014,329-342$

ISSN: 1311-8080 (printed version); ISSN: 1314-3395 (on-line version)

url: http://www.ijpam.eu

doi: http://dx.doi.org/10.12732/ijpam.v96i3.4

\title{
AG CODES AND VECTOR BUNDLES ON RATIONAL SURFACES
}

\author{
Tohru Nakashima \\ Department of Mathematical and Physical Sciences \\ Japan Women's University \\ Mejirodai 2-8-1, Bunkyoku, Tokyo, JAPAN
}

\begin{abstract}
We prove the existence of weakly stable bundles on a curve on certain rational surfaces defined over a finite field. As an application, we give certain bounds of the parameters of the AG codes constructed from these bundles.
\end{abstract}

AMS Subject Classification: 94B27, 14H60, 14J60

Key Words: algebraic-geometric codes, vector bundles

\section{Introduction}

Let $\mathbb{F}_{q}$ be the finite field with $q$ elements and let $X$ be a projective variety defined over $\mathbb{F}_{q}$, which is smooth and geometrically irreducible. Let $\mathcal{P}=$ $\left\{P_{1}, P_{2}, \ldots, P_{n}\right\}$ be a set of distinct $\mathbb{F}_{q}$-rational points of $X$. Let $L$ be a line bundle on $X$ defined over $\mathbb{F}_{q}$. The algebraic-geometric(AG) code is defined to be the image of the evaluation map

$$
\begin{aligned}
\varphi: H^{0}(X, L) & \rightarrow \bigoplus_{i=1}^{n} L_{P_{i}} \cong \mathbb{F}_{q}^{n} . \\
f & \mapsto\left(f\left(P_{1}\right), f\left(P_{2}\right), \ldots, f\left(P_{n}\right)\right)
\end{aligned}
$$

Received: May 10, 2014

(c) 2014 Academic Publications, Ltd. url: www.acadpubl.eu 
Recently, several constructions of the AG codes from vector bundles of higher rank have been proposed.

The first example is the scroll codes, which is obtained by taking $X=\mathbb{P}(E)$, the projective bundle associated to a vector bundle $E$ on a curve in the above construction.

In $[2],[10]$, the minimum distances of the scroll codes have been studied and in [5],[7] higher weights have been investigated. The second example is the code introduced by Savin by evaluating sections of a vector bundle at finite number of rational points. It turned out that the problem of determining the parameters of these codes defined by vector bundles is related to certain stability properties. It has been shown that we may give lower bounds for the minimum distance using weakly stable bundles([11],[13]). One way to construct weakly stable bundles is restricting a stable bundle on a variety containing the curve. Exploiting a theorem of Langer, we proved in [12] that a stable bundle on a smooth projective surface restricts to a weakly stable bundle on curves of sufficiently large degree. The restriction theorem, together with the existence result of stable bundles on the projective plane $\mathbb{P}^{2}$, allows us to construct AG codes of Savin type from vector bundles on plane curves.

In this paper we discuss the existence of weakly stable bundles on curves on certain rational surfaces and its application to the construction of AG codes.

In Section 2 we prove a restriction theorem for rank two bundles on a surface, which is an improvement of the result obtained in [12]. The proof of this result is based on a theorem of Hein $([4])$.

We consider in Section 3 the existence of stable bundles on Hirzebruch surfaces and del-Pezzo surfaces defined over finite fields using the extension method. Although the existence of stable bundles has been extensively studied in the case of algebraically closed fields, very few results are known over finite fields. It would be interesting to consider the existence problem for more general surfaces.

Section 4 is devoted to the study of AG codes from vector bundles. Our improved restriction theorem allows us to estimate the parameters of the scroll codes on curves on rational surfaces. We also construct AG codes of Savin type on curves in $\mathbb{P}^{1} \times \mathbb{P}^{1}$.

\section{Weakly Stable Bundles via Restriction}

In this section we prove a restriction theorem for stable bundles on surfaces. For properties of vector bundles on surfaces, we refer the reader to [6]. 
Let $k$ be an algebraically closed field of characteristic $p>0$. Let $X$ be a smooth and irreducible projective curve defined over $k$ and let $E$ be a vector bundle of rank $r$ on $X$. The slope of $E$ is defined by

$$
\mu(E)=\frac{\operatorname{deg} E}{r} .
$$

For a rational number $\alpha, E$ is said to be weakly stable of type $\alpha$ if, for any sub-line bundle $L \subset E$, we have

$$
\mu(L) \leq \mu(E)+\alpha .
$$

We recall the method of constructing weakly stable bundles of type $\alpha$ via restriction of stable bundles on a surface as discussed in [12]. Let $S$ be a smooth, irreducible projective surface defined over $k$. A polarized surface is a pair $(S, H)$ of smooth, irreducible projective surface $S$ and a very ample line bundle $H$ on it.

For any line bundles $L, M$ on $S$, let $L \cdot M$ denote their intersection number. Let $E$ be a torsion-free sheaf of rank $r$ and the $i$-th Chern classes $c_{i}(E)=c_{i}$ on $S$. The discriminant $\Delta(E)$ of $E$ is defined by

$$
\Delta(E)=2 r c_{2}(E)-(r-1) c_{1}(E)^{2} .
$$

For an ample line bundle $H$ on $S$, the $H$-slope of $E$ is defined by

$$
\mu_{H}(E)=\frac{c_{1}(E) \cdot H}{r} .
$$

If $X \subset S$ is a smooth irreducible curve and $E$ is a vector bundle on $S$, the restriction $E_{\mid X}$ to $X$ is a vector bundle of rank $r$ and degree $c_{1}(E) \cdot X$ on $X$.

A torsion-free sheaf $E$ of rank $r$ on $S$ is said to be $H$-stable if, for any coherent subsheaf $F \subset E$ with $0<\operatorname{rk} F<r$, we have

$$
\mu_{H}(F)<\mu_{H}(E) .
$$

We are interested in the problem whether an $H$-stable bundle $E$ on $S$ restricts to a weakly stable bundle $E_{\mid X}$ on $X \subset S$.

We choose a nef divisor $A$ on $S$ such that $T_{S}(A)$ is globally generated and let $\beta_{r}$ be the number defined by

$$
\beta_{r}=\left(\frac{r(r-1)}{p-1} A \cdot H\right)^{2} .
$$

Using the restriction theorem of Langer([8]), we have the following result $([12$, Proposition 4.1]). 
Proposition 2.1. Let $(S, H)$ be a polarized surface defined over $k$. Let $E$ be an $H$-stable vector bundle of rank $r$ on $S$. Let $\alpha$ be a rational number and let $l$ be an integer with

$$
l \geq \frac{r-1}{r H^{2}}\left(H^{2} \Delta(E)-2 r H^{2} \alpha+\frac{1}{(r-1)^{2}}+2 r(r-1) \beta_{r}\right) .
$$

Then, for any smooth irreducible curve $X \in|l H|$, the restriction $E_{\mid X}$ of $E$ to $X$ is a vector bundle which is weakly stable of type $\alpha$.

We shall show that a stronger restriction theorem holds for rank two bundles. We define the minimal $H$-degree $a$ is defined as follows.

$$
a=\min \{L \cdot H>0 \mid L \in \operatorname{Pic}(S)\} .
$$

A line bundle $L$ on $S$ is said to be $H$-minimal if $L \cdot H=a$.

Following [4], we define the number $\beta$ by

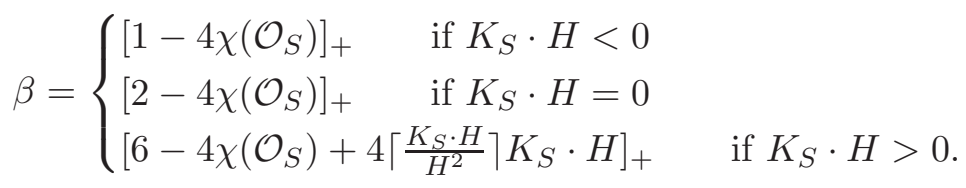

Here we set $[x]_{+}=\max \{x, 0\}$.

Let $X \subset S$ a smooth irreducible curve. Let $E$ be a rank two bundle on $S$. If there exists a surjection $E_{\mid X} \rightarrow T$ to a line bundle $T$ on $X$. Then the kernel $G$ of the composite map $E \rightarrow E_{\mid X} \rightarrow T$ is locally free and said to be the elementary transformation of $E$ along $T$. Then $G$ fits in the exact sequence

$$
0 \rightarrow G \rightarrow E \rightarrow T \rightarrow 0 .
$$

Lemma 2.2. Let $S$ be a smooth and irreducible projective surface defined over $k$ and let $E$ be a rank two bundle on $S$. Let $X \subset S$ be a smooth irreducible curve and let $T$ be a line bundle of degree $\gamma$ on $X$. Assume that

$$
X^{2}>\Delta(E)+\gamma-\beta .
$$

Let $G$ denote the elementary transformation of $E$ along $T$. Then there exists a sub-line bundle $A \subset G$ with the following property: for any ample line bundle $H, B:=2 A-c_{1}(G)-X$ satisfies the inequality

$$
(B \cdot H)^{2} \geq H^{2}(-\Delta(E)-2 B \cdot X-\gamma) .
$$


Proof.

$$
\begin{aligned}
\Delta(G) & =\Delta(E)-X^{2}+2\left(2 \operatorname{deg} T-c_{1}(E) \cdot X\right) \\
& <\Delta(E)-H^{2} l^{2}-4 \alpha .
\end{aligned}
$$

By assumption on $l$, we have $\Delta(G)<\beta$. Hence Corollary 2.6 of [4] implies that $G$ is Bogomolov unstable, that is, there exists a line bundle $A$ such that $G$ can be written as an extension

$$
0 \rightarrow A \rightarrow G \rightarrow \mathcal{I}_{Z}\left(c_{1}(G)-A\right) \rightarrow 0
$$

such that $c_{1}(G)-2 A$ belongs to the positive cone of $S$. Thus we have $\left(c_{1}(G)-\right.$ $2 A) \cdot H \geq 0$ for any ample line bundle $H$ and $\left(c_{1}(G)-2 A\right)^{2}>0$.

We have $l(Z)=c_{2}(G(-A))=c_{2}(G)-c_{1}(G) \cdot A+A^{2} \geq 0$. Hence $4 A^{2}-$ $4 c_{1}(G) \cdot A \geq-4 c_{2}(G)$. Thus, setting $B=2 A-c_{1}(G)-X$, we have

$$
\begin{aligned}
B^{2} & =4 A^{2}-4 c_{1}(G) \cdot A+c_{1}(G) 2-4 A \cdot X+2 c_{1}(G) \cdot X+X^{2} \\
& \geq-4 c_{2}(G)+c_{1}(G) 2-4 A \cdot X+2 c_{1}(G) \cdot X+X^{2} \\
& =-\Delta(G)-2 B \cdot X-X^{2} \\
& =-\Delta(E)-2 B \cdot X-\gamma .
\end{aligned}
$$

On the other hand, by the Hodge index theorem, we have $(B \cdot H)^{2} \geq H^{2} B^{2}$. This yields $(B \cdot H)^{2} \geq H^{2}(-\Delta(E)-2 B \cdot X-\gamma)$ as desired.

The following is a slight modification of a theorem of Hein([4]).

Theorem 2.3. Let $(S, H)$ be a polarized surface defined over $k$. Let $E$ be an $H$-stable vector bundle of rank two on $S$. Let $\alpha<0$ be a rational number and let $l$ be an integer with

$$
l>\max \left\{\frac{a}{2 H^{2}}+\frac{\Delta(E)}{2 a}, \quad \sqrt{\frac{\Delta(E)-4 \alpha-\beta}{H^{2}}}\right\} .
$$

Then, for any smooth irreducible curve $X \in|l H|$, the restriction $E_{\mid X}$ of $E$ to $X$ is weakly stable of type $\alpha$.

Proof. Assume that there exists a smooth and irreducible curve $X \in|l H|$ such that $E_{\mid X}$ is not weakly stable of type $\alpha$. Then, there exists a sub-line bundle $L \subset E_{\mid X}$ satisfying $\operatorname{deg} L>\mu\left(E_{\mid X}\right)+\alpha$. Setting $T=E_{\mid X} / L$, we have $\operatorname{deg} T=c_{1}(E) \cdot X-\operatorname{deg} L$. Hence

$$
2 \operatorname{deg} T-c_{1}(E) \cdot X<-2 \alpha .
$$


Let $G$ denote the elementary transformation of $E$ along $T$. By the lemma above, there exists a sub-line bundle $A \subset G$ such that $B=2 A-c_{1}(G)-l H$ satisfies the inequality

$$
l^{2}(B \cdot H)^{2} \geq H^{2}(-2 l B \cdot H-\Delta(E)-\gamma) .
$$

Setting $f(x):=l^{2} x^{2}+2 H^{2} l x+H^{2}(\Delta(E)+\gamma)$, this yields $f(B \cdot H) \geq 0$. Since the discriminant $D=l^{2}\left(H^{2}\right)^{2}-\Delta(E) H^{2}$ of $f(x)$ is positive by assumption, the quadratic equation $f(x)=0$ has two distinct solution $-l H^{2} \pm \sqrt{D}$. Since $-l H^{2}-\sqrt{D}<B \cdot H$, we have

$$
B \cdot H \geq-l H^{2}+\sqrt{D} .
$$

By the assumption on $l$, we obtain $B \cdot H>-a$. Hence we have $B \cdot H \geq 0$, otherwise we would have $-B \cdot H<a$ which contradicts the definition of $a$. Thus we see that $A$ is a subsheaf of $E$ satisfying $A \cdot H \geq \mu_{H}(E)$ which is impossible. This completes the proof.

Remark 2.4. If we set $r=2$ in Proposition 2.1, we obtain the bound

$$
l \geq \frac{1}{2 H^{2}}\left(H^{2} \Delta(E)-4 H^{2} \alpha+1+4 \beta_{2}\right) .
$$

The right-hand side grows like $O(-\alpha)$ as $\alpha$ goes to $-\infty$ while Theorem 2.3 above gives a better bound of order $O(\sqrt{-\alpha})$.

\section{Existence of Stable Bundles on Rational Surfaces}

In this section we prove the existence of weakly stable bundles on curves in certain rational surface defined over finite fields.

Let $X$ be a smooth and geometrically irreducible projective curve defined over $\mathbb{F}_{q}$ and $E$ a vector bundle on $X$ defined over $\mathbb{F}_{q}$. Let $\bar{X}=X \times \overline{\mathbb{F}}_{q}$ and let $\bar{E}$ be the base change of $E$ to $\bar{X}$, where $\overline{\mathbb{F}}_{q}$ denotes the algebraic closure of $\mathbb{F}_{q}$. We say that $E$ is weakly stable or weakly stable of type $\alpha$ if so is $\bar{E}$. Similarly, if $(S, H)$ is a pair of a smooth, geometrically irreducible projective surface and a very ample line bundle on it defined over $\mathbb{F}_{q}$, then we say that $(S, H)$ is a polarized surface if so is $(\bar{S}, \bar{H})$. A vector bundle $E$ on $S$ is said to be $H$-stable if $\bar{E}$ is $\bar{H}$-stable.

For a nonnegative integer $n$, let $S=F_{n}$ be a Hirzebruch surface defined over $\mathbb{F}_{q}$. That is, $F_{n}$ is the projective bundle $\mathbb{P}(\mathcal{E})$ associated to the rank two 
bundle $\mathcal{E}=\mathcal{O}_{\mathbb{P}^{1}} \oplus \mathcal{O}_{\mathbb{P}^{1}}(-n)$ over $\mathbb{P}^{1}$. Let $\Sigma=\mathcal{O}(1)$ denote the tautological line bundle on $S$ and let $f$ denote the class of a fiber over a $\mathbb{F}_{q^{-}}$-rational point of $\mathbb{P}^{1}$. The canonical bundle of $S$ is given by $K_{S}=-2 \Sigma-(n+2) f$. A line bundle $H=\Sigma+m f$ is a very ample if and only if $m>n$ which we fix in what follows.

Next we consider del-Pezzo surfaces. Assume that $q>3$. Let $P_{1}, P_{2}, \ldots, P_{m}$

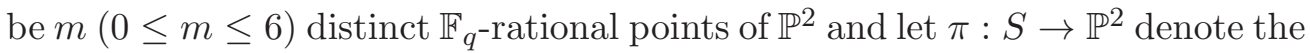
blow-up at these points. Let $E_{i}$ be the exceptional divisor over $P_{i}$ and let $\mathcal{O}_{\mathbb{P}^{2}}(1)$ be the hyperplane bundle on $\mathbb{P}^{2}$. We assume that $P_{i}$ are in general position, that is, no three of them are colinear and no six of them are contained in a quadric. Then the canonical bundle of $S$ is given by $K_{S}=\pi^{*} \mathcal{O}_{\mathbb{P}^{2}}(-3)-\sum_{i=1}^{m} E_{i}$. We fix a very ample line bundle $H=\pi^{*} \mathcal{O}_{\mathbb{P}^{2}}(3)-\sum_{i=1}^{m} E_{i}$.

Lemma 3.1. Let $(S, H)$ be a Hirzebruch surface or a del-Pezzo surface as above. For any $c>0$, there exists a 0 -dimensional closed subscheme $Z \subset S$ of length $c$ defined over $\mathbb{F}_{q}$, whose support consists of $c$ distinct points.

Proof. Assume that $S$ is a Hirzebruch surface. For any $c>0$, we have $\# S\left(\mathbb{F}_{q^{c}}\right)=\left(q^{c}+1\right)^{2}=q^{2 c}+2 q^{c}+1$. Hence there exists a point $P \in\left(S\left(\mathbb{F}_{q^{c}}\right) \backslash\right.$ $\cup_{j<c} S\left(\mathbb{F}_{q^{j}}\right)$. Let $F: \overline{\mathbb{F}}_{n} \rightarrow \overline{\mathbb{F}}_{n}$ denote the Frobenius map and put $Z:=P+$ $F(P)+F^{2}(P)+\cdots+F^{c-1}(P)$. By construction, $Z$ consists of $c$ distinct points and is defined over $\mathbb{F}_{q}$ since $Z$ is invariant under $F$. Similarly, in the case of del-Pezzo surface $S$, for any $c>0$, we have $\# S\left(\mathbb{F}_{q^{c}}\right)=q^{2 c}+2\left(q^{c}+1\right)-m$. Hence we can proceed as before.

If $(S, H)$ be a Hirzebruch surface or a del-Pezzo surface as above, then we have $a=1$. Indeed, the line bundles $L=\Sigma+(1+n-m) f$ and $L=E_{i}$ are $H$ minimal since $L \cdot H=1$. In what follows we fix these $H$-minimal line bundles. Further, we have $\beta=0$ since $K_{S} \cdot H<0$ in both cases.

We have the following result concerning the existence of $H$-stable bundles on these rational surfaces.

Proposition 3.2. Let $(S, H)$ be a Hirzebruch surface or a del-Pezzo surface and let $L$ be the line bundle as above. For all integers $r \geq 2$ and all $c \geq r$, there exists an $H$-stable bundle $E_{r, c}$ on $S$ defined over $\mathbb{F}_{q}$, of rank $r, c_{1}\left(E_{r, c}\right)=L$ and $c_{2}\left(E_{r, c}\right)=c$. Further we have $h^{0}\left(E_{r, c}\right) \geq r-1$ and $\operatorname{dim} \operatorname{Ext}^{1}\left(E_{r, c}, \mathcal{O}_{S}\right)=c-r+1$.

Proof. We prove the claim by induction on $r$. By Lemma 3.1, for any $c \geq 1$, we may choose a 0 -dimensional subscheme $Z$ of length $c$ defined over $\mathbb{F}_{q}$ such that the support consists of $c$ distinct points. Then the ideal sheaf $\mathcal{I}_{Z}$ is defined over $\mathbb{F}_{q}$ and $c_{2}\left(\mathcal{I}_{Z}\right)=c$. Since we have $\left|L+K_{S}\right|=\emptyset$, by Serre 
correspondence([11, Lemma 3.2]), there exists a rank two bundle $E_{2, c}$ which fits in a non-split extension

$$
0 \rightarrow \mathcal{O}_{S} \rightarrow E_{2, c} \rightarrow \mathcal{I}_{Z}(L) \rightarrow 0 .
$$

Then $E_{2, c}$ is defined over $\mathbb{F}_{q}$ and $h^{0}\left(E_{2, c}\right) \geq 1$. Since $L$ is $H$-minimal, $E$ is $H$-stable by [9, Lemma 1.4]. By the exact sequence

$$
0 \rightarrow I_{Z}\left(L+K_{S}\right) \rightarrow \mathcal{O}_{S}\left(L+K_{S}\right) \rightarrow \mathcal{O}_{Z}\left(L+K_{S}\right) \rightarrow 0,
$$

the induced cohomology sequence gives $\operatorname{Ext}^{1}\left(\mathcal{I}_{Z}(L), \mathcal{O}_{S}\right) \cong H^{1}\left(\mathcal{I}_{Z}\left(L+K_{S}\right)\right) \cong$ $H^{0}\left(\mathcal{O}_{Z}\left(L+K_{S}\right)\right) \cong \mathbb{F}_{q}^{\oplus c}$. Hence $\operatorname{dim} \operatorname{Ext}^{1}\left(E_{2, c}, \mathcal{O}_{F_{n}}\right)=c-1$ and the claim is true for $r=2$.

Assume that the claim holds up to $r-1$. Then, for any $c \geq r-1$, there exists an $H$-stable bundle $E_{r-1, c}$ satisfying $h^{0}\left(E_{r-1, c}\right) \geq r-2$ and $\operatorname{dim} \operatorname{Ext}^{1}\left(E_{r-1, c}, \mathcal{O}_{S}\right)=$ $c-r+2$. In particular, for any $c \geq r$, we have $\operatorname{dim} \operatorname{Ext}^{1}\left(E_{r-1, c}, \mathcal{O}_{S}\right)>0$. Hence there exists a non-split extension

$$
0 \rightarrow \mathcal{O}_{S} \rightarrow E_{r, c} \rightarrow E_{r-1, c} \rightarrow 0 .
$$

The bundle $E_{r, c}$ is $H$-stable as before. Considering the induced cohomology sequence, we obtain $h^{0}\left(E_{r, c}\right)=h^{0}\left(E_{r-1, c}\right)+1 \geq r-1$ and $\operatorname{dim} \operatorname{Ext}^{1}\left(E_{r, c}, \mathcal{O}_{S}\right)=$ $\operatorname{dim} \operatorname{Ext}^{1}\left(E_{r-1, c}, \mathcal{O}_{S}\right)-1=c-r+1$. Hence the claim is true for $r$. This completes the proof.

By Theorem 2.3 and Proposition 3.2, we obtain the following

Proposition 3.3. Let $(S, H)$ be a Hirzebruch surface or a del-Pezzo surface defined over $\mathbb{F}_{q}$. Let

$$
l_{0}:=\max \left\{\frac{1}{2(2 m-n)}+2 m-n+6, \quad \sqrt{\frac{2 m-n+6-4 \alpha}{2 m-n}}\right\}
$$

if $(S, H)$ is a Hirzebruch surface and let

$$
l_{0}:=\max \left\{\frac{1}{2(9-m)}+\frac{9}{2}, \quad \sqrt{\frac{9-4 \alpha}{9-m}}\right\}
$$

if $(S, H)$ is a del-Pezzo surface. Let $X \in|l H|$ be a smooth and geometrically irreducible curve defined over $\mathbb{F}_{q}$. If $l>l_{0}$, then there exists a rank two bundle $E$ on $X$ of degree $l$ which is weakly stable of type $\alpha$. 
Proof. Let $\widetilde{E}:=E_{2,2}$ in Proposition 3.2. Then $\Delta(\widetilde{E})=2 m-n+6$ if $(S, H)$ is a Hirzebruch surface(resp. $\Delta(\widetilde{E})=9$ if $(S, H)$ is a del-Pezzo surface). By Theorem 2.3, $E:=\widetilde{E}_{\mid X}$ for $X \in|l H|$ for $l>l_{0}$ is the desired bundle.

\section{Application to AG Codes}

In this section we consider the two types of AG codes defined from vector bundles defined over $\mathbb{F}_{q}$ where $q=p^{m}$ for a prime $p$.

Let $X$ be a projective curve defined over $\mathbb{F}_{q}$, which is smooth and geometrically irreducible. For a vector bundle $E$ on $X$ defined over $\mathbb{F}_{q}$, let $\pi: Y:=$ $\mathbb{P}(E) \rightarrow X$ denote the associated projective bundle. Let $\mathcal{P}=\left\{P_{1}, P_{2}, \ldots, P_{n}\right\}$ be a set of distinct $\mathbb{F}_{q}$-rational points of $Y$. For a point $P_{i} \in \mathcal{P}$, let $\kappa\left(P_{i}\right)$ denote the residue field at $P_{i}$. For a line bundle $L$ on $Y$ defined over $\mathbb{F}_{q}$, For each $i$ we fix an isomorphism $L_{P_{i}}:=L \otimes \kappa\left(P_{i}\right) \cong \mathbb{F}_{q}$. The scroll code $C(Y, \mathcal{P}, L)$ is defined to be the image of the evaluation map

$$
\begin{aligned}
\varphi: H^{0}(Y, L) & \rightarrow \bigoplus_{i=1}^{n} L_{P_{i}} \cong \mathbb{F}_{q}^{n} \\
s \quad & \mapsto\left(s\left(P_{1}\right), s\left(P_{2}\right), \ldots, s\left(P_{n}\right)\right) .
\end{aligned}
$$

We simply write $C(Y, L)$ if $\mathcal{P}=Y\left(\mathbb{F}_{q}\right)$.

For any rank two bundle $E$ on $X$, let $L_{\max }$ be a maximal sub-line bundle of $E$, that is, $L_{\max }=\max _{L \subset E} \operatorname{deg} L$ where $L$ runs through all sub-line bundles of $E$. First, we define the Segre invariant $s_{1}(E)$ of $E$ as the following integer:

$$
s_{1}(E)=\operatorname{deg} E-2 \operatorname{deg} L_{\max } .
$$

We also recall the invariant $e$ as defined in $[3, \mathrm{Ch} . \mathrm{V}]$. A rank two bundle $E$ is said to be normalized if $H^{0}(E) \neq 0$ and, for all line bundle $M$ with $\operatorname{deg} M<0$, we have $H^{0}(E(M))=0$. Then we define $e(E)=-\operatorname{deg} E$ for such $E$.

Lemma 4.1. Let $E$ be a rank two bundle which is weakly stable of type $\alpha<0$ and let $E_{\text {norm }}:=E \otimes L_{\max }^{\vee}$ for a maximal sub-line bundle $L_{\max }$. Then we have

$$
e\left(E_{\text {norm }}\right) \leq 2 \alpha \text { and } \mu\left(E_{\text {norm }}\right) \geq-\alpha .
$$

Proof. If $E$ is weakly stable of type $\alpha$, then we have $\operatorname{deg} L_{\max } \leq \mu(E)+\alpha$ and hence $\alpha \geq \frac{s_{1}(E)}{2}$. On the other hand, we see that $E_{\text {norm }}$ is normalized. Indeed, the injection $L_{\max } \hookrightarrow E$ yields a non-trivial section of $E_{\text {norm. }}$. Let 
$M$ be a line bundle with $\operatorname{deg} M<0$, then we have $H^{0}\left(E_{\text {norm }}(M)\right)=0$. For, otherwise we would have a non-trivial map $L_{\max } \otimes M^{\vee} \rightarrow E$, which contradicts the definition of $L_{\max }$. Since $e\left(E_{\text {norm }}\right)=-\operatorname{deg} E_{\text {norm }}=-s_{1}(E)$, the claim follows.

We have the following result for the parameters of scroll codes obtained from rank two vector bundles on rational surfaces. We notice that similar results have been obtained by Ballico in the case of plane curves([1]).

Theorem 4.2. Let $(S, H)$ be a Hirzebruch surface or a del-Pezzo surface defined over $\mathbb{F}_{q}$. For a given rational number $\alpha<0$, let $l$ be an integer with $l>l_{0}$ where $l_{0}$ is as in Proposition 3.3. Let $X \in|l H|$ be a smooth and geometrically irreducible curve of genus $g(X)$ defined over $\mathbb{F}_{q}$ with $n_{0}=\# X\left(\mathbb{F}_{q}\right)$. For nonnegative integers $b_{1}, b_{2}$, let $N=\left(\alpha+\frac{g(X)-1}{p}\right) b_{1}+b_{2}$. Assume that

$$
n_{0}>N, \quad b_{1} \leq p-1 \quad \text { and } \quad-\alpha b_{1}+b_{2}>2 g(X)-2 .
$$

Then there exists a ruled surface $\pi: Y \rightarrow X$ such that the line bundle $L \equiv$ $b_{1} \mathcal{O}_{Y}(1)+b_{2} f$ on $Y$ yields an $[n, k, d]$-code $C(Y, L)$ with

$$
\begin{aligned}
& n=(q+1) n_{0}, \\
& k \geq\left(b_{1}+1\right)\left\{-\alpha b_{1}+b_{2}+1-g(X)\right\}, \\
& d \geq\left(q+\left(1-b_{1}\right)\right)\left(n_{0}-N\right) .
\end{aligned}
$$

Here $\mathcal{O}_{Y}(1)$ denotes the tautological line bundle on $Y$ and $f$ the class of a $\pi$-fiber.

Proof. Under the assumptions, there exists a rank two bundle $E$ which is weakly stable bundle of type $\alpha$ on $X$. Let $Y=\mathbb{P}\left(E_{\text {norm }}\right)$. By [10, Theorem 3.1], we obtain estimates for the parameters of the AG codes defined by the line bundle $L$ on $Y$.

Remark 4.3. By the adjunction formula, for any smooth curve $X \in|l H|$, $g(X)$ can be computed as follows.

$$
g(X)=\frac{\left(l H+K_{S}\right) \cdot l H}{2}+1 .
$$

Further, the Hasse-Weil theorem yields

$$
\# X\left(\mathbb{F}_{q}\right) \geq q+1-2 g(X) \sqrt{q} .
$$


Thus we have

$$
\begin{aligned}
g(X) & =\frac{l^{2}(2 m-n)+l(n-2 m-2)}{2}+1, \\
n_{0} & \geq q+1-\left\{l^{2}(2 m-n)+l(n-2 m-2)+2\right\} \sqrt{q}
\end{aligned}
$$

if $(S, H)$ is a Hirzebruch surface and

$$
\begin{aligned}
g(X) & =\frac{l^{2}(9-m)+l(m-9)}{2}+1, \\
n_{0} & \geq q+1-\left\{l^{2}(9-m)+l(m-9)+2\right\} \sqrt{q}
\end{aligned}
$$

if $(S, H)$ is a del-Pezzo surface.

Next we consider the AG codes of Savin type. Let $X$ be a projective curve defined over $\mathbb{F}_{q}$, which is smooth and geometrically irreducible. Let $E$ be a vector bundle of rank $r$ on $X$ defined over $\mathbb{F}_{q}$. For a set $\mathcal{P}=\left\{P_{1}, P_{2}, \ldots, P_{n}\right\}$ of distinct $\mathbb{F}_{q^{-}}$rational points of $X$, we fix isomorphisms $E_{P_{i}} \cong \mathbb{F}_{q}^{r} \cong \mathbb{F}_{Q}^{n}$ as $\mathbb{F}_{q^{-}}$ vector spaces where we set $Q=q^{r}$. Let

$$
\begin{aligned}
& \varphi: H^{0}(X, E) \rightarrow \bigoplus_{i=1}^{n} E_{P_{i}} \cong \mathbb{F}_{Q}^{n} \\
& s \quad \mapsto\left(s\left(P_{1}\right), s\left(P_{2}\right), \ldots, s\left(P_{n}\right)\right)
\end{aligned}
$$

be the evaluation map. We define the code $C(X, \mathcal{P}, E)$ to be the image of $\varphi$ considered as an $\mathbb{F}_{Q}$-vector space. In case $\mathcal{P}=X\left(\mathbb{F}_{q}\right)$, we write $C(X, \mathcal{P}, E)=$ $C(X, E)$. It should be noted that although $C(X, \mathcal{P}, E)$ is an $\mathbb{F}_{q}$-subspace of $\mathbb{F}_{Q}^{n}$, it is not an $\mathbb{F}_{Q^{-}}$subspace in general.

The length $n$ of $C(X, \mathcal{P}, E)$ is clearly equal to $\# \mathcal{P}$ and the dimension $k$ is given by the real number

$$
k=\log _{q^{r}} \# C(X, \mathcal{P}, E) .
$$

Since $C(X, \mathcal{P}, E)$ is $\mathbb{F}_{q}$-linear subspace of $\mathbb{F}_{Q}^{n}$, the minimum distance $d$ is given by

$$
d=\min _{c \neq 0 \in C(X, \mathcal{P}, E)} w(c)
$$

where $w(c)$ denotes the weight of $c$.

We have the following bounds for the parameters of $C(X, \mathcal{P}, E)([11$, Proposition 2.3]). 
Lemma 4.4. If $E$ is weakly stable of type $\alpha$ and $n>\mu(E)+\alpha$, then $C(X, \mathcal{P}, E)$ has the following parameters.

$$
\begin{aligned}
& k \geq \frac{h^{0}(X, E)}{r}, \\
& d \geq n-\mu(E)-\alpha .
\end{aligned}
$$

In $[12$, Theorem 5.4] we estimated the parameters of the codes $C(X, E)$ obtained from a bundle $E$ on a plane curve $X$. Now we consider the case of curves on $F_{0}=\mathbb{P}^{1} \times \mathbb{P}^{1}$.

Proposition 4.5. Let $(S, H)=\left(F_{0}, \Sigma+m f\right)$ over $\mathbb{F}_{q}$. Let $X \in|l H|$ be a curve on $S$ defined over $\mathbb{F}_{q}$, which is smooth and geometrically irreducible. Let $n=\# X\left(\mathbb{F}_{q}\right)$. Let $r \geq 2$ be an integer and $\alpha<0$ a rational number. Assume that

$$
l>\frac{r-1}{r}\left(2 r^{2}-2(r-1)(1-m)-2 r \alpha+\frac{1}{2 m(r-1)^{2}}\right)
$$

and

$$
n>\frac{l}{r}+\alpha \text {. }
$$

Then there exists a vector bundle $E$ of rank $r$ on $X$ which defines the code $C(X, E)$ with the parameters

$$
\begin{aligned}
& k \geq \frac{r-1}{r}, \\
& d \geq n-\frac{l}{r}-\alpha .
\end{aligned}
$$

Proof. Let $\widetilde{E}:=E_{r, r}$ as in Proposition 3.2. Since $T_{S}$ is globally generated, we may choose $\beta_{r}=0$ for all $r \geq 2$ in Proposition 2.1 . Hence $E:=\widetilde{E}_{\mid X}$ is a weakly stable bundle of type $\alpha$. By Lemma 4.4 and [12, Lemma 4.2], we obtain the following bounds on the parameters of $C(X, E)$.

$$
\begin{aligned}
& k \geq \frac{h^{0}(S, \widetilde{E})}{r} \geq \frac{h^{0}(X, E)}{r} \geq \frac{r-1}{r}, \\
& d \geq n-\mu(E)-\alpha=n-\frac{l}{r}-\alpha .
\end{aligned}
$$

Remark 4.6. By [11, Remark 1], Savin's algorithm can correct all $\epsilon$ errors with

$$
\epsilon<\left\lfloor t^{*}-\frac{\alpha+m l^{2}-(m+1) l+1}{2}\right\rfloor
$$


where $t^{*}$ denotes the designed correction capacity

$$
t^{*}=\left\lfloor\frac{n-\frac{l}{r}}{2}\right\rfloor .
$$

\section{Acknowledgments}

The author was supported in part by Grant-in-Aid for Scientific Research (C)(24540052).

\section{References}

[1] E.Ballico, Scroll codes over curves of higher genus: reducible and superstable vector bundles, Des.Codes Cryptogr. 63, No.3 (2012), 365-377. DOI:10.1007/s10623-011-9561-9

[2] S.H.Hansen, Error-correcting codes from higher dimensional varieties, Finite Fields Appl. 7, No.4 (2001), 530-552. DOI:10.1006/ffta.2001.0313

[3] R.Hartshorne, Algebraic Geometry, Springer Verlag, Berlin (1977).

[4] G.Hein, Restriction of stable rank two vector bundles in arbitrary characteristic, Commun.in Alg. 34, No.7 (2006), 2319-2335. DOI:10.1080/00927870600550145

[5] G.M.Hana, T.Johnsen, Scroll codes, Des.Codes Cryptogr. 45, No.3 (2007), 365-377. DOI:10.1007/s10623-007-9131-0

[6] D.Huybrechts, M.Lehn, The Gometry of Moduli Spaces of Sheaves, Second Ed., Cambridge University Press, Cambridge (2010).

[7] T.Johnsen, N.H.Rasmussen, Scroll codes over curves of higher genus, Appl.Algebra Eng.Commun.Comput. 21, No.3 (2010), 397-415. DOI:10.1007/s00200-010-0130-4

[8] A.Langer, Semistable sheaves in positive characteristic, Ann. of Math. 159, No.1 (2004), 251-276. DOI:10.4007/annals.2004.159.251

[9] T.Nakashima, Reflection of sheaves on a Calabi-Yau variety, Asian J.Math. 6, No.3 (2002), 567-577. 
[10] T.Nakashima, Error-correcting codes on projective bundles, Finite Fields Appl. 12, No.2 (2006), 222-231. DOI:10.1016/j.ffa.2005.04.008

[11] T.Nakashima, AG codes from vector bundles, Des. Codes Cryptogr. 57, No.1 (2010), 107-115. DOI:10.1007/s10623-009-9354-3

[12] T.Nakashima, Parameters of $A G$ codes from vector bundles, Finite Fields and Their Appl. 18, No.4 (2012), 746-759. DOI:10.1016/j.ffa.2012.04.004

[13] V.Savin, Algebraic-geometric codes from vector bundles and their decoding, arxiv: math/08031096 